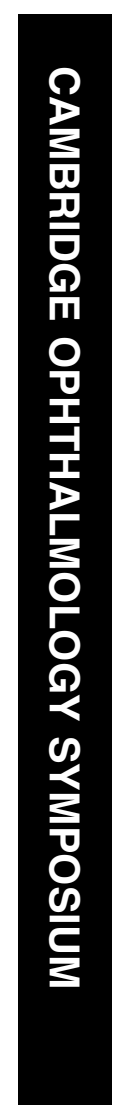

\section{Improving RPE adhesion to Bruch's membrane}

\begin{abstract}
Age-related macular degeneration is the leading cause of blindness in the developing world. Retinal pigmented epithelium (RPE) transplantation in subretinal space, has been assessed in various animal models of age-related macular degeneration and in humans as a potential technique to preserve the visual function. However, the RPE cell survival posttransplantation is limited because of lack of attachment of the transplanted cells to the pathological Bruch's membrane and also partly because of iatrogenic removal of adhesive elements in the membrane during the removal of choroidal new vessels before transplantation procedure. Although pathological Bruch's membrane is well studied, there is still much debate as to why and how changes in the structure and components of this membrane leads to loss of RPE cells and disruption of their function and subsequent death of photoreceptors leading to visual loss. Integrins on RPE cells have been characterized and shown to be important for attachment of cells to Bruch's membrane. Considering the essential role of integrins in functions such as cell migration and adhesion, it is plausible that lack of attachment of RPE cells posttransplantation can be overcome by improving integrin function. Here, we have focused on some of the recent findings on the use of integrins and modulation of their function to improve the adhesion of RPE cells to normal and pathological Bruch's membrane. This work also aims at elucidating a potential mechanism by which accumulating inhibitory molecules in the Bruch's membrane in the pathological state, interferes with integrin function.
\end{abstract}

Eye (2009) 23, 1890-1893; doi:10.1038/eye.2008.411; published online 16 January 2009

the 2008 Cambridge Ophthalmological review). Symposium (requested
FT Afshari and JW Fawcett

macular degeneration; choroidal

neovascularization

\section{Introduction}

Age-related macular degeneration (ARMD) is classically divided into dry atrophic type with formation of drusen between Bruch's membrane and the retinal pigmented epithelium (RPE) cell layer and a wet exudative type with formation and growth of choroidal new vessels (CNVs), which invade and leak fluid into the subretinal space. The exudative type of this condition is almost always associated with severe loss of vision. Although our understanding of the two types has increased substantially, there is still much controversy as to how the disease progresses and what elements lead to progression from dry to the wet type of ARMD.

The retinal pigmented epithelial cells be involved in preserving photoreceptors and their function. One of their major functions is to remove the shed outer segments of the photoreceptors, ${ }^{1,2}$ and if they fail there is buildup of debris and retinal degeneration. ${ }^{3}$ A focus in the study of the pathology of ARMD is Bruch's membrane and the changes that take place between the inner collagenous zone of this membrane and RPE cell layer. Abnormalities in Bruch's membrane in ARMD lead to disruption of RPE cell layer function ${ }^{4}$ and this in turn leads to disruption of photoreceptor function and their loss. There are therefore research efforts to preserve the RPE layer and to replace it if it is damaged. Various techniques have been used to prevent the visual loss in macular degeneration ranging from cell transplantation ${ }^{5-7}$ to the use of anti-VEGF antibodies to prevent formation of new vessels ${ }^{8,9}$ and halt the progress of the disease.

RPE transplantation has been used in various animal models of ARMD and has been shown to
Keywords: retinal pigmented epithelium; Bruch's membrane; integrin; age-related 
be able to prevent continuing loss of vision in the context of macular degeneration. One of the techniques used in human patients has been removal of CNVs from the abnormally neovascularized Brush's membrane, followed by autologous transplantation of RPE cells subretinally to allow repopulation of the Bruch's membrane. ${ }^{10,11}$ Although this technique is effective in removing the CNVs, an undesirable consequence is the stripping of RPE cells and removal of the basement membrane of Bruch's exposing the deeper collagenous layers. However, functional recovery post-RPE transplantation has been shown to be limited because most of the RPE cells die shortly after transplantation, and they do not migrate out from the site of implantation. Lack of attachment and adhesion of RPE cells after transplantation has been shown to be the major cause of death, because the cells are unable to acquire the adhesive signals necessary to avoid cell death ${ }^{12,13}$ and to initiate migration.

\section{Bruch's membrane and RPE attachment}

The aging process is one of the most important risk factors in macular degeneration. Bruch's membrane is a pentalaminar structure with each layer containing different extracellular ligands. Recent studies from various groups have shown clearly that human aged Bruch's membrane supports the attachment of RPE cells to a lesser extent than membranes from young donors. ${ }^{14}$ In addition analysis of RPE adhesion to various components and layers of Bruch's membrane has revealed that not all layers of this membrane support adhesion to the same extent with the uppermost basement membrane, to which the RPE cells normally adhere, being one of the most supporting layers. ${ }^{15}$ Therefore, it is plausible that pathological processes, which lead to accumulation of various substrates including inhibitory antiadhesive molecules in the membrane and also removal of supporting layers postsurgically will have adverse effects on the adhesion of RPE cells. The damaged Bruch's membrane found in AMRD, particularly the wet form, shows many of the molecular changes typical of damaged tissue, with upregulation of antiadhesive molecules such as tenascin-C. ${ }^{16,17}$

\section{Integrins and their role in adhesion}

Integrins are transmembrane heterodimer structures, whose function is to mediate interactions between cells and extracellular matrix molecules. They are involved in many cellular functions such as cell adhesion, migration, proliferation, differentiation, and cell survival. They are heterodimers, composed of an $\alpha$-subunit and a $\beta$-subunit and dimersation is necessary for the function of the integrin. Depending on the combination of $\alpha$ - and $\beta$-subunit, integrin dimers can bind to various extracellular ligands present in Bruch's membrane to promote adhesion of the cells the basement membrane. Various integrin subunits have been shown to be expressed by RPE cells, ${ }^{18}$ potentially allowing their interaction with laminin, fibronectin, vitronectin, and collagen within the lamina of membrane. There is a substantial body of literature demonstrating the importance of integrins in many of the functions of RPE cells. Various groups, including our own, have asked whether manipulation of RPE integrins might be a way to improve their survival posttransplantation and to improve the performance and healing ability of endogenous cells. Study of RPE integrins should also increase our understanding of RPE-Bruch's dynamics in normal and pathological state.

Primary uncultured RPE cells have been shown to have low levels of integrins on their cell surface, but they can be upregulated by prolonged cell culture. It has been shown that increasing the expression of integrins on the cell surface by long-term culturing of the primary RPE cells before transplantation is one way to improve the adhesion to the Bruch's membrane by allowing better engagement with the extracellular ligands available in the pathological Bruch's membrane. ${ }^{19}$ This partially prevents cell loss immediately after transplantation, providing a feasible practical approach in tackling the adhesion problem.

An alternative approach to the same problem of low levels of surface integrin has been use of gene delivery techniques to overexpress integrins in RPE cells to further support their attachment. This was shown by Fang et $a l^{20}$ recently who demonstrated that increasing $\alpha 6-$ and $\beta 4$-integrin improved the adhesion of RPE cells to Bruch's membrane and its components.

\section{Modulation of integrin function and role of inhibitory molecules}

Although expression of integrins by cells is important, they must be placed on the cell surface, activated, and linked to both the cytoskeleton and signalling pathways to be effective. This means that there are several possible ways to fine tune and control integrin function.

One approach to improve adhesion to the Bruch's membrane, which is a focus of our lab, involves modulating the integrin affinity state; this is termed integrin activation. Although the presence of integrin on the cell surface is necessary for engagement with the ligands, the affinity and activation state of the integrins also is an important factor in cell behaviour. Integrins switch between an activated high affinity state and a 
deactivated low affinity state, modulating the level of adhesion and intracellular signalling. Integrin activation has been shown to be developmentally modulated ${ }^{21}$ and also various inhibitory factors have been shown to modulate the integrin activation state and their ligandbinding affinity. ${ }^{22,23}$ The integrin activation state therefore acts as to fine tune their function allowing control of cell adhesion and signalling. Unpublished work from our lab shows that integrin activation in RPE cells improves both the adhesion and migration rate of RPE cells on the ECM ligands that exist within the Bruch's membrane. Forced activation of integrins also allows a higher level of adhesion to the Bruch's membrane isolated from adult rat eyes. Although use of integrin activation on normal substrates improves attachment, the affinity state of integrins is likely to be even more crucial where RPE cells have to interact with an abnormal Bruch's membrane expressing various inhibitory molecules in addition to the proadhesive molecules such as laminin and fibronectin. Transplanted primary RPE cells (which have only a small amount of integrin on their cell surface) will probably benefit from a higher quantity of cell surface integrin in order that they can adhere to the pathological nonadhesive Bruch's membrane. In addition, integrin activation increases the affinity of the integrins for their specific ligands so manipulation of the activation state may also be useful in RPE cells. The extracellular matrix contains a mixture of adhesive and repulsive molecules, and after injury there is an increase in repulsive molecules such as chondroitin sulphate proteoglycans and tenascin. On the basis of recent work in our laboratory, integrin activation using various agents can overcome the antiadhesive effect of proteoglycans. These molecules build up in Bruch's membrane as a result of neovascularization and fibrosis.

Another technique to improve RPE survival and their adhesion is to express integrins that can engage with the inhibitory ligands in the pathological Bruch's membrane. RPE cells lack or substantially downregulate several integrins in the adult state and are therefore unable to overcome the accumulating inhibitory substrates that are found in ARMD, which can act as an antiadhesive cues interfering with the integrin function of RPE. On the basis of unpublished work from our lab, expression of integrins capable of interacting with inhibitory ligands such as tenascin, which are present in pathological membrane allows improved adhesion without adversely affecting the attachment to the positive cues.

Overall, integrin engineering in RPE cells, by increasing the expression level of integrins that recognize molecules present in the pathological Bruch's membrane, and by increasing their affinity for their ligands by activation may be the key to overcoming the inhibitory substrates in the Bruch's membrane. In transplanted cells, this may allow RPE cells to engage with the membrane after transplantation and to survive and migrate, allowing these cells to integrate and repopulate the subretinal space.

\section{Acknowledgements}

This work was supported by Sim's Scholarship (F T Afshari).

\section{References}

1 Finnemann SC, Silverstein RL. Differential roles of CD36 and alphavbeta 5 integrin in photoreceptor phagocytosis by the retinal pigment epithelium. J Exp Med 2001; 194: 1289-1298.

2 Chang Y, Finnemann SC. Tetraspanin CD81 is required for the alpha $\mathrm{v}$ beta5-integrin-dependent particle-binding step of RPE phagocytosis. J Cell Sci 2007; 120: 3053-3063.

3 Nandrot EF, Kim Y, Brodie SE, Huang X, Sheppard D, Finnemann SC. Loss of synchronized retinal phagocytosis and age-related blindness in mice lacking alphavbeta5 integrin. J Exp Med 2004; 200: 1539-1545.

4 Sun K, Cai H, Tezel TH, Paik D, Gaillard ER, Del Priore LV. Bruch's membrane aging decreases phagocytosis of outer segments by retinal pigment epithelium. Mol Vis 2007; 13: 2310-2319.

5 Gouras P, Algvere P. Retinal cell transplantation in the macula: new techniques. Vision Res 1996; 36: 4121-4125.

6 MacLaren RE, Uppal GS, Balaggan KS, Tufail A, Munro PMG, Milliken AB et al. Autologous transplantation of the retinal pigment epithelium and choroid in the treatment of neovascular age-related macular degeneration. Ophthalmology 2007; 114: 561-570.

7 Peyman GA, Blinder KJ, Paris CL, Alturki W, Nelson Jr NC, Desai U. A technique for retinal pigment epithelium transplantation for age-related macular degeneration secondary to extensive subfoveal scarring. Ophthalmic Surg 1991; 22: 102-108.

8 Bopp S. Anti-VEGF for neovascular ARMD: visual improvement as the goal of therapy? Br J Ophthalmol 2007; 91: $1259-1260$.

9 Wu L, Martinez-Castellanos MA, Quiroz-Mercado H, Arevalo JF, Berrocal MH, Farah ME et al. Twelve-month safety of intravitreal injections of bevacizumab (Avastin(R)): results of the Pan-American Collaborative Retina Study Group (PACORES). Graefes Arch Clin Exp Ophthalmol 2008; 246: 81-87.

10 Treumer F, Bunse A, Klatt C, Roider J. Autologous retinal pigment epithelium-choroid sheet transplantation in age related macular degeneration: morphological and functional results. Br J Ophthalmol 2007; 91: 349-353.

11 Bindewald A, Roth F, Van Meurs J, Holz FG. Transplantation of retinal pigment epithelium (RPE) following CNV removal in patients with AMD. Techniques, results, outlook. Ophthalmologe 2004; 101: 886-894.

12 Tezel TH, Del Priore LV. Reattachment to a substrate prevents apoptosis of human retinal pigment epithelium. Graefes Arch Clin Exp Ophthalmol 1997; 235: 41-47. 
13 Tezel TH, Del Priore LV. Repopulation of different layers of host human Bruch's membrane by retinal pigment epithelial cell grafts. Invest Ophthalmol Vis Sci 1999; 40: 767-774.

14 Gullapalli VK, Sugino IK, Van Patten Y, Shah S, Zarbin MA. Impaired RPE survival on aged submacular human Bruch's membrane. Exp Eye Res 2005; 80: 235-248.

15 Del Priore LV, Tezel TH. Reattachment rate of human retinal pigment epithelium to layers of human Bruch's membrane. Arch Ophthalmol 1998; 116: 335-341.

16 Fasler-Kan E, Wunderlich K, Hildebrand P, Flammer J, Meyer P. Activated STAT 3 in choroidal neovascular membranes of patients with age-related macular degeneration. Ophthalmologica 2005; 219: 214-221.

17 Nicolo M, Piccolino FC, Zardi L, Giovannini A, Mariotti C. Detection of tenascin-C in surgically excised choroidal neovascular membranes. Graefes Arch Clin Exp Ophthalmol 2000; 238: 107-111.

18 Zarbin MA. Analysis of retinal pigment epithelium integrin expression and adhesion to aged submacular human Bruch's membrane. Trans Am Ophthalmol Soc 2003; 101: 499-520.
19 Gullapalli VK, Sugino IK, Zarbin MA. Culture-induced increase in alpha integrin subunit expression in retinal pigment epithelium is important for improved resurfacing of aged human Bruch's membrane. Exp Eye Res 2008; 86: 189-200.

20 Fang IM, Yang CH, Yang CM, Chen MS. Overexpression of integrin alpha(6) and beta(4) enhances adhesion and proliferation of human retinal pigment epithelial cells on layers of porcine Bruch's membrane. Exp Eye Res 2008; 88: 12-21.

21 Ivins JK, Yurchenco PD, Lander AD. Regulation of neurite outgrowth by integrin activation. J Neurosci 2000; 20 : 6551-6560.

22 Bourgin C, Murai KK, Richter M, Pasquale EB. The EphA4 receptor regulates dendritic spine remodeling by affecting beta1-integrin signaling pathways. J Cell Biol 2007; 178: 1295-1307.

23 Miao H, Burnett E, Kinch M, Simon E, Wang B. Activation of EphA2 kinase suppresses integrin function and causes focal-adhesion-kinase dephosphorylation. Nat Cell Biol 2000; 2: 62-69. 